\title{
Posttranslational Processing of Secretory Component in the Rat Jejunum by a Brush Border Metalloprotease
}

\author{
D. J. Ahnen, J. R. Singleton, T. C. Hoops, and T. M. Kloppel \\ Veterans Administration Medical Center; and Departments of Medicine; and Biochemistry, Biophysics and Genetics, University of \\ Colorado School of Medicine, Denver, Colorado 80220
}

\begin{abstract}
Secretory component (SC) is a glycoprotein that mediates the transcellular transport of polymeric immunoglobulins into external secretions. SC is synthesized and inserted into the plasma membrane of epithelial cells and hepatocytes as a transmembrane protein, where it serves as a receptor for polymeric immunoglobulins. SC is posttranslationally cleaved to a soluble protein before secretion into external fluids. In the rat jejunum, we observed that the molecular weights of both the major membrane and soluble forms of SC were 10,000-20,000 smaller than the comparable hepatic forms of the glycoprotein. We therefore set out to determine the reason for the differences in size of SC between these two tissues. The smaller size of jejunal SC was not due to the action of pancreatic proteases or differential glycosylation but was due to proteolysis by a jejunal brush border protease. The protease was characterized as a metalloprotease, with a pH optimum of $\sim 5$. It is present in jejunal, ileal, and renal tubular brush borders as an integral membrane constituent. When the protease was inhibited in vivo, conversion of jejunal secretory component to the smaller size was partially prevented. Thus, in the rat jejunum, SC undergoes two posttranslational proteolytic events: conversion of membrane secretory component to the soluble form and conversion of soluble SC to a smaller size by a previously undescribed brush border protease.
\end{abstract}

\section{Introduction}

Secretory component $(\mathrm{SC})^{1}$ is a glycoprotein that is synthesized and secreted by epithelial cells in the intestine (1-4), mammary gland (5-7), and salivary glands as well as by the hepatocyte of the rat $(8,9)$. SC is synthesized as an integral membrane protein (mSC) that is inserted into the abluminal plasma membrane (laterobasal membrane of epithelial cells; sinusoidal membrane of hepatocytes) where it serves as the receptor for polymeric immunoglobulins (pIg). pIg binds to $\mathrm{mSC}$, and the $\mathrm{mSC}-\mathrm{pIg}$

Portions of this work have appeared in abstract form, 1984. J. Cell Biol. 99(4, Pt. 2):281 $a$; and 1985. Gastroenterology. 88:1422.

Address reprint requests to Dr. Ahnen, Veterans Administration Medical Center 111E, 1055 Clermont St., Denver, CO 80220.

Received for publication 1 July 1985.

1. Abbreviations used in this paper: BB, brush border membranes; DTT, dithiothreitol; LBM, laterobasal membranes; 2-ME, 2 mercaptoethanol; mSC, membrane secretory component; pIg, polymeric immunoglobulins; PMSF, phenylmethylsulfonyl fluoride; SC, secretory component; SDS, sodium dodecyl sulfate; SDS-PAGE, SDS-polyacrylamide gel electrophoresis; sIg, secretory immunoglobulins; TFMS, trifluoromethane sulfonic acid.

The Journal of Clinical Investigation, Inc. Volume 77, June 1986, 1841-1848 complex is internalized into a vesicle and transported to the luminal surface of the cell where the complex is secreted (1012). The transcellular transport and secretion of SC also occurs in the absence of pIg; consequently, both soluble SC-pIg complexes (secretory immunoglobulin; sIg) and uncomplexed soluble SC (free SC) are present in many external secretions.

sIg and free SC may enter the lumen of the intestine either via the bile or by transport across the enterocyte. While many investigators have examined SC biosynthesis and processing in the hepatocyte $(8,9)$ or mammary gland $(5-7)$, little is known about the processing of SC in the intestinal cell. We report that in the rat jejunum SC is secreted in a molecular form that is smaller than the SC observed in bile and other glandular secretions. The smaller SC is produced by a proteolytic step mediated by an integral endopeptidase of the jejunal brush border (BB).

\section{Methods}

Animals. Male Sprague-Dawley rats (Charles River Breeding Laboratories, Inc., Wilmington, MA) weighing 250-300 g, were used for all experiments. Animals were fasted for $18 \mathrm{~h}$ before each experiment. Animals were anesthetized with sodium pentobarbital $(50 \mathrm{mg} / \mathrm{kg})$, injected intraperitoneally. Except where noted, all samples of intestinal fluid, bile, and membranes were collected or prepared in the presence of $1 \mathrm{mM}$ phenylmethylsulfonyl fluoride (PMSF) and $50 \mu \mathrm{g} / \mathrm{ml}$ soybean trypsin inhibitor.

Antisera. Monospecific antibody to rat SC was prepared in goats by immunization with SC purified from rat bile as previously described (8). Rabbit anti-goat IgG conjugated to horseradish peroxidase was purchased from Cooper Biomedical, Inc. (Malvern, PA).

Biological material. Rat liver membranes were prepared by acetone precipitation and $1 \%$ sodium dodecyl sulfate (SDS) extraction as previously described (8). Bile was collected from the proximal common bile duct via a polyethylene cannula (PE10; Clay Adams Div., Becton, Dickinson \& Co., Parsippany, NJ) into tubes on ice. The bile was collected either into Laemmli sample buffer (8) containing $1 \mathrm{mM}$ PMSF and 50 $\mu \mathrm{g} / \mathrm{ml}$ soybean trypsin inhibitor for immediate SDS-polyacrylamide gel electrophoretic (SDS-PAGE) analysis, or into $150 \mathrm{mM} \mathrm{NaCl}, 1 \mathrm{mM}$ PMSF, $50 \mu \mathrm{g} / \mathrm{ml}$ soybean trypsin inhibitor for storage at $-20^{\circ} \mathrm{C}$ and subsequent study.

Jejunal and colonic secretions were collected from isolated loops of gut from 18-h fasted, anesthetized animals. A $25-\mathrm{cm}$ section of jejunum beginning at the ligament of Treitz, or of the colon from the cecum to rectum, was isolated and cannulated at both ends. The loop was flushed with $150 \mathrm{mM} \mathrm{NaCl}$ to remove luminal contents, then perfused with 150 $\mathrm{mM} \mathrm{NaCl}$ for $1 \mathrm{~h}$ to remove adherent biliary and pancreatic proteases. The loop was perfused for $5 \mathrm{~min}$ with $150 \mathrm{mM} \mathrm{NaCl}$ containing or lacking protease inhibition ( $1 \mathrm{mM}$ PMSF, $50 \mu \mathrm{g} / \mathrm{ml}$ soybean trypsin inhibitor), then clamped at both ends. After $1 \mathrm{~h}, 2-3 \mathrm{ml}$ of intestinal fluid was aspirated from the loop and centrifuged $(13,000 \mathrm{~g}$ for $2 \mathrm{~min}$ ) to remove cells and debris. The supernatant was mixed either with Laemmli sample buffer containing protease inhibitors ( $1 \mathrm{mM}$ PMSF, $50 \mu \mathrm{g} /$ $\mathrm{ml}$ soybean trypsin inhibitor) for immediate SDS-PAGE analysis or with protease inhibitors ( $1 \mathrm{mM}$ PMSF, $50 \mu \mathrm{g} / \mathrm{ml}$ soybean trypsin inhibitor) for storage at $-20^{\circ} \mathrm{C}$. In some experiments, intestinal secretions were collected with additional protection against proteolysis. That is, $150 \mathrm{mM}$ 2-mercaptoethanol (2-ME) was used in place of $150 \mathrm{mM} \mathrm{NaCl}$ for per- 
fusion of the loop, and EDTA at a final concentration of $50 \mathrm{mM}$ was added to the secretions.

Jejunal laterobasal membranes (LBM), Golgi membranes, lysosomes, and BB were prepared from mucosal scrapings of jejunal loops. LBM and Golgi membranes were isolated by density gradient ultracentrifugation as previously described (10). Jejunal, ileal, and renal tubular BB were prepared by $\mathrm{Mg}^{++}$precipitation (11). Lysosomal membranes were prepared by density gradient centrifugation (11), using beta glucosaminidase as the marker enzyme. NaK-ATPase, galactosyl transferase, and sucrase activities were used as markers for LBM, Golgi, and BB, respectively (11). Enrichment, yield, and recovery of the enzyme were calculated as previously described (11). In some experiments, jejunal membranes were rapidly prepared in the presence of additional protease inhibitors; isolated jejunal loops were perfused with $150 \mathrm{mM}$ 2-ME, $1 \mathrm{mM}$ PMSF, and $50 \mu \mathrm{g} / \mathrm{ml}$ soybean trypsin inhibitor for $1 \mathrm{~h}$, then flushed with 150 $\mathrm{mM}$ 2-ME, and $50 \mathrm{mM}$ EDTA. Mucosal scrapings were homogenized (Polytron homogenizer; Brinkmann Instruments Co., Westbury, NY; setting 6; $30 \mathrm{sec}$, two times) in $150 \mathrm{mM}$ 2-ME, $50 \mathrm{mM}$ EDTA, $1 \mathrm{mM}$ PMSF, and $50 \mu \mathrm{g} / \mathrm{ml}$ soybean trypsin inhibitor. The homogenate was centrifuged ( $750 \mathrm{~g}$ for $10 \mathrm{~min}$ ) to remove cells and nuclei. Membranes were pelleted from the supernate by centrifugation $(20,000 \mathrm{~g}$ for $20 \mathrm{~min})$, suspended, and boiled ( $3 \mathrm{~min}$ ) in Laemmli sample buffer for SDS-PAGE and anti-SC immunoblot analysis.

Rat milk was collected from lactating Sprague-Dawley mothers 3$14 \mathrm{~d}$ after delivery. After administration of light pentobarbital anesthesia and oxytocin $\left(0.03-0.05 \mathrm{~cm}^{3} / 100 \mathrm{~g}\right.$ body weight), milk was collected by suction with light vacuum directly into an equal volume of $1 \mathrm{mM}$ PMSF and $50 \mu \mathrm{g} / \mathrm{ml}$ soybean trypsin inhibitor. The whole milk was immediately ultracentrifuged $(100,000 \mathrm{~g}$ for $30 \mathrm{~min})$, and the whey was drawn off from below the fatty layer. The whey was dialyzed against $\mathrm{pH} 6$ buffer at $4^{\circ} \mathrm{C}$ for $2 \mathrm{~h}$ and recentrifuged $(10,000 \mathrm{~g}$ for $20 \mathrm{~min})$ to remove the precipitated casein. The supernatant was mixed with an equal amount of Laemmli sample buffer containing $1 \mathrm{mM} \mathrm{PMSF}$ and $50 \mu \mathrm{g} / \mathrm{ml}$ soybean trypsin inhibitor and stored at $4^{\circ} \mathrm{C}$.

Long-term isolated jejunal loops. In order to collect secretions from jejunal loops that were totally free of pancreatic secretions, long-term isolated loops of jejunum were prepared. Fasted $200-300-\mathrm{g}$ rats were anesthetized, and a $10-15-\mathrm{cm}$ section of jejunum distal to the ligament of Treitz was isolated, leaving the mesenteric vasculature intact. Both ends of the isolated loops were brought through incisions in the abdominal wall and anastomosed to the skin. Intestinal continuity was reestablished by a jejuno-jejunostomy. The loops were flushed twice weekly with saline. $4 \mathrm{wk}$ after surgery, secretions from the loops were collected $1 \mathrm{~h}$ after filling the loops with $3 \mathrm{ml}$ of $150 \mathrm{mM} \mathrm{NaCl}, 1 \mathrm{mM} \mathrm{PMSF}$, and $50 \mu \mathrm{g} /$ $\mathrm{ml}$ soybean trypsin inhibitor.

Trifluoromethane sulfonic acid (TFMS) deglycosylation. Samples of rat bile, small intestinal secretions, and bovine serum albumin (a nonglycosylated protein) were treated with TFMS, using a modification of a described technique (12). Bile and jejunal secretions were dialyzed against water to remove salts and then lyophilized. TFMS and anisole were mixed 2:1; and $1 \mathrm{ml}$ of this solution was added to $10-\mathrm{mg}$ samples of lyophilized bile, jejunal secretions, or albumin. After $30 \mathrm{~s}$ of dehydration with nitrogen, samples were allowed to react for intervals of 30 , 90 , and $180 \mathrm{~min}$ at $0^{\circ} \mathrm{C}$ with constant stirring. The reaction was quenched with a 35 -fold excess of diethyl ether in $10 \% n$-hexane, and the samples were incubated at $-70^{\circ} \mathrm{C}$ for $1 \mathrm{~h}$ to precipitate proteins. If after $20 \mathrm{~min}$ no precipitate was observed, a drop of pyridine was added to cause coprecipitation of sample proteins with the pyridine methane sulfonic salts. The precipitated proteins were pelleted by centrifugation $(1500 \mathrm{~g}$ for 10 min), washed twice in ether, and dissolved in $5 \mathrm{ml}$ of $\mathrm{H}_{2} \mathrm{O}$ by vigorous vortexing. After centrifugation ( $4000 \mathrm{~g}$ for $10 \mathrm{~min}$ ), the supernatant was dialyzed against $\mathrm{H}_{2} \mathrm{O}$ and lyophilized. The lyophilized samples were resuspended in Laemmli sample buffer containing protease inhibitors for SDS-PAGE analysis.

In vitro mixing of bile and $B B$ membranes. $50 \mu \mathrm{l}$ of bile was mixed with $50 \mu \mathrm{g}$ of $\mathrm{BB}$ and incubated at $20^{\circ} \mathrm{C}$ for $30 \mathrm{~min}$. The mixtures were diluted in Laemmli sample buffer and prepared for SDS-PAGE and antiSC immunoblotting.
After observing that biliary $\mathrm{SC}$ was hydrolyzed to a smaller size upon incubation with BB, we partially characterized the SC hydrolytic activity. The $\mathrm{pH}$ dependence of the hydrolase was examined by carrying out identical BB-bile incubations over a pH range of 4-10. To determine if the hydrolytic activity was an integral or peripheral protein of the BB, jejunal BB were extracted with $0.5 \%$ Triton X-100 or $0.5 \mathrm{M} \mathrm{KCl}$ for $1 \mathrm{~h}$ at $0^{\circ} \mathrm{C}$ and centrifuged $(13,000 \mathrm{~g}$ for $20 \mathrm{~min})$. The supernates and pellets were incubated with bile $\left(20^{\circ} \mathrm{C}\right.$ for $\left.30 \mathrm{~min}\right)$, and the mixtures were analyzed by SDS-PAGE. To further characterize the hydrolytic activity, BB-bile incubations were conducted in the presence of the following protease inhibitors: $1 \mathrm{mM}$ tosyl-lysine-chloromethylketone; $1 \mathrm{mM}$ tosylamide-phenyl-ethylchloromethylketone; $1 \mathrm{mM}$ disiopropylfluorophosphate; $25 \mu \mathrm{g} / \mathrm{ml}$ pepstatin; $1 \mathrm{mM}$ p-chloromercuriphenyl sulfonic acid; $1 \mathrm{mM}$ iodoacetamide; $2 \mathrm{mM}$ dithiothreitol (DTT); $100 \mathrm{mM} 2 \mathrm{ME}$; $2 \mathrm{mM}$ EDTA; $40 \mu \mathrm{g} / \mathrm{ml}$ phosphoramidon (only the 2-ME, EDTA, and DTT led to inhibition of the hydrolytic activity). To examine the metal ion dependence of the EDTA inhibition, BB were washed in $50 \mathrm{mM}$ EDTA three times, then washed in $150 \mathrm{mM} \mathrm{NaCl}$ three times to remove the EDTA. BB-bile incubations were also carried out in the presence or absence of a variety of divalent metal cations $(\mathrm{Ca}, \mathrm{Mg}, \mathrm{Zn}, \mathrm{Mn}, \mathrm{Co})$ at concentrations ranging from $10^{-3}$ to $10^{-6} \mathrm{M}$.

SDS-PAGE and anti-SC immunoblots. Samples were electrophoretically separated in $8.5 \%$ polyacrylamide slab gels using an S-600 apparatus (Hoefer Scientific Instruments, San Francisco, CA) as previously described $(8,13)$. An aliquot of each sample was stained with Coomassie Blue R250; an identical sample was run on the same gel for immunoblotting of SC. Immunoblots were performed as previously described (8), using goat anti-rat SC followed by horseradish-peroxidase-conjugated rabbit anti-goat IgG. Molecular weight markers (Bio-Rad Laboratories, Richmond, CA) were used on each gel. Since the amount of SC present in the membranes and secretions varied greatly between the organs studied, the amount of protein loaded onto each lane of a given gel was optimized to allow adequate visualization of the immunoreactive SC bands.

Processing of biliary SC by the small intestine. In order to characterize the fate of biliary SC in the intestine, bile containing biosynthetically labeled biliary proteins was injected into an isolated loop of small intestine. The bile had been obtained from the proximal bile duct of another anesthetized rat after an intraperitoneal injection of $1 \mathrm{mCi}$ of $\left[{ }^{3} \mathrm{H}\right]$ fucose (Amersham Corp., Arlington Heights, IL). Aliquots of the radiolabeled bile were then injected into loops of jejunum that had been isolated in vivo in a separate rat and prepared as described above for collection of jejunal secretions. After a 1-h incubation, the small intestinal contents were collected from the isolated loop, diluted in Laemmli sample buffer, and analyzed by SDS-PAGE and fluorography. To eliminate the effect of pancreatic proteases on biliary SC, parallel experiments were performed in isolated jejunal loops that had been pretreated by perfusion with 150 $\mathrm{mM} \mathrm{NaCl}$, containing $1 \mathrm{mM} \mathrm{PMSF}$ and $50 \mu \mathrm{g} / \mathrm{ml}$ soybean trypsin inhibitor for $1 \mathrm{~h}$ before injection of radiolabeled bile. To differentiate between the effects of jejunal secretions and brush border constituents on biliary SC, aliquots of radiolabeled bile were incubated with jejunal secretions or saline for $1 \mathrm{~h}$ at $37^{\circ} \mathrm{C}$ in the presence or absence of $1 \mathrm{mM}$ PMSF and $50 \mu \mathrm{g} / \mathrm{ml}$ soybean trypsin inhibitor.

\section{Results}

Molecular forms of SC. The molecular forms of SC in bile, milk, jejunal secretions, and colonic secretions were identified by immunoblots. Bile, milk, and colonic secretions contained an SC doublet (82,000 and 78,000 molecular weight [mol wt]), whereas jejunal secretions contained a smaller form of SC that was predominantly a single 70,000-mol-wt band (Fig. 1). To determine if a smaller size of SC was also present in jejunal membranes, anti-SC immunoblots of jejunal LBM and liver membranes were performed (Fig. 2; membrane forms). In liver membranes, SC was present as a doublet $(110,000$ and $100,000 \mathrm{~mol} \mathrm{wt})$ whereas in jejunal LBM, a single 90,000-mol-wt SC band was found. Thus, the membrane form, as well as the secreted form of SC 


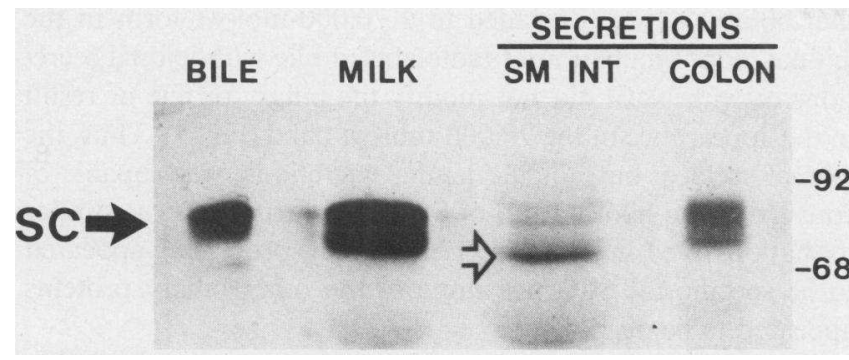

Figure 1. Molecular forms of SC in rat bile, milk, small intestinal (SM INT) and colonic secretions. Rat bile, milk, small intestinal and colonic secretions were collected in the presence of pancreatic protease inhibitors ( $1 \mathrm{mM}$ PMSF and $50 \mu \mathrm{g} / \mathrm{ml}$ soybean trypsin inhibitor) and subjected to SDS-PAGE and anti-SC immunoblots. Whereas SC in bile, milk, and colonic secretions was an 80,000-mol-wt doublet (solid arrow), the major SC band in small intestinal secretions was a 70,000mol-wt singlet (open arrow).

in the jejunum, was $10,000-20,000$ mol wt smaller than SC prepared in a similar manner from the liver (Fig. 2).

When secretions were collected from loops of jejunum that had been isolated from pancreatic secretions for $4 \mathrm{wk}$, a single 70,000-mol-wt form of SC was present (Fig. 3). This SC was identical in size to that present in jejunal secretions collected from intact jejunal loops and smaller than biliary SC. Thus, the smaller size of jejunal SC was not due to the action of pancreatic proteases.

TFMS deglycosylation. In order to determine if jejunal SC was smaller than biliary SC because of differential glycosylation, bile and jejunal secretions were subjected to TFMS-mediated deglycosylation and immunoblotting for SC (Fig. 4). Although originally reported to remove $O$-linked sugars, TFMS deglycosylation of $N$-linked carbohydrates has been subsequently dem-

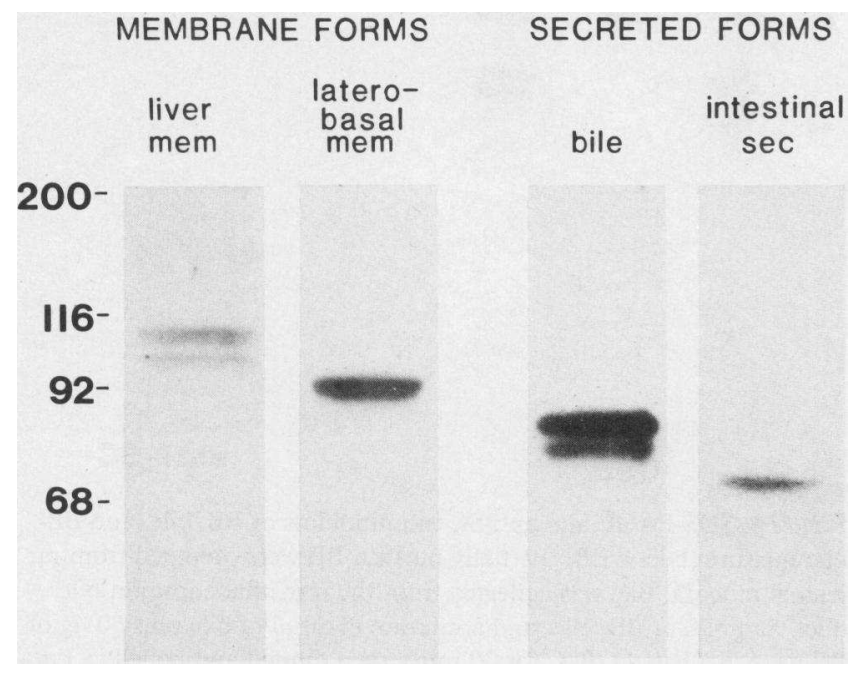

Figure 2. Molecular forms of SC in membranes and secretions of the liver and small intestine. Liver membranes, jejunal LBM, bile, and small intestinal secretions were prepared or collected in the presence of pancreatic protease inhibitors ( $1 \mathrm{mM} \mathrm{PMSF}$ and $50 \mu \mathrm{g} / \mathrm{ml}$ soybean trypsin inhibitor) and subjected to SDS-PAGE and anti-SC immunoblots. In the small intestine, both the membrane (laterobasal mem) and secreted (intestinal sec) forms of SC were single bands that were about 10,000-20,000 mol wt smaller than the doublets present in comparable preparations from the liver (liver membrane and bile).

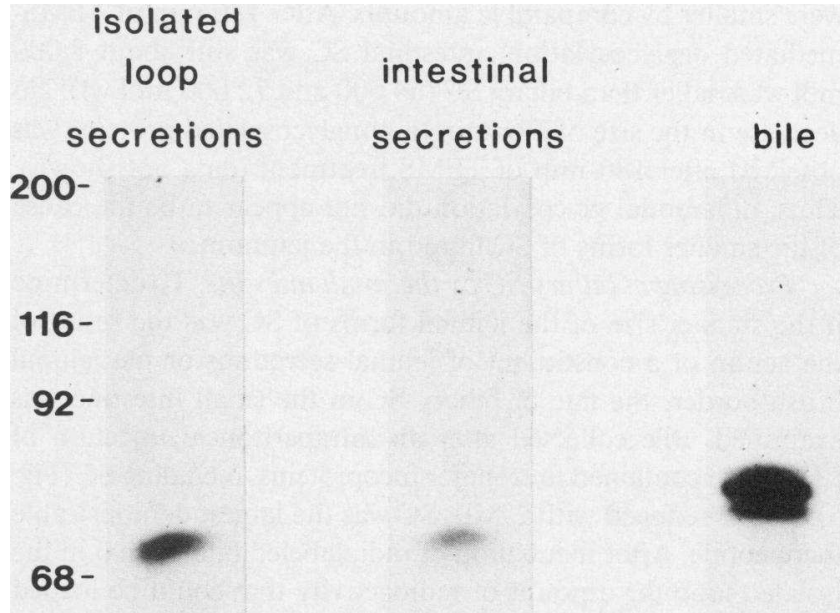

Figure 3. Molecular forms of SC in secretions from long-term isolated jejunal loops. Intestinal secretions were collected from loops of jejunum that had been isolated from pancreatic secretions in vivo for 4 wk (see text for surgical details), and were subjected to SDS-PAGE and anti-SC immunoblots. Secretions from the long-term isolated jejunal loops contained SC that was identical in size to that found in intestinal secretions from an intact jejunal loop (intestinal secretions) and about $10,000 \mathrm{~mol} w \mathrm{wt}$ smaller than the SC doublet found in bile.

onstrated (14). After 30 min of incubation with TFMS, SC in both the bile and jejunal secretions was decreased by about 7,000 mol wt. Similarly, after 90 and $180 \mathrm{~min}, \mathrm{SC}$ from both secretions

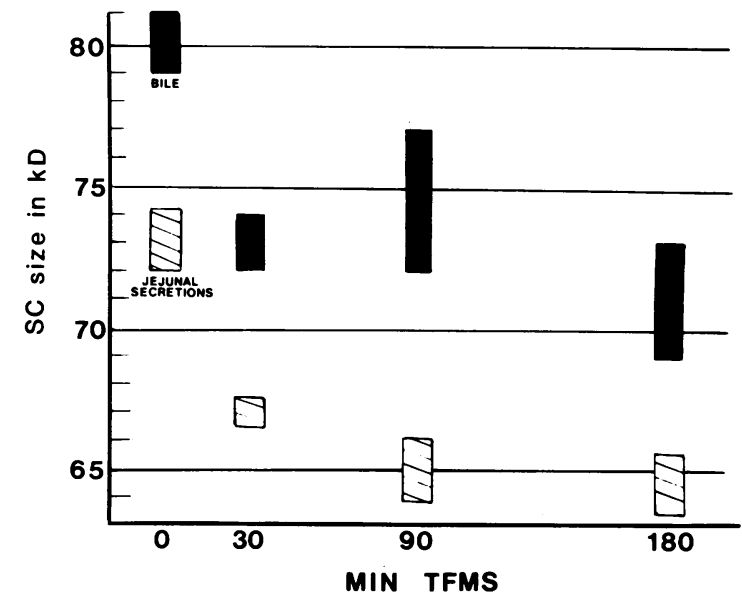

Figure 4. Deglycosylation of SC from bile and intestinal secretions with TFMS. $1 \mathrm{ml}$ of TFMS in anisole (2:1) was added to $10 \mathrm{mg}$ of lyophilized bile or small intestinal secretions. After $0,30,90$, or 180 min of incubation at $0^{\circ} \mathrm{C}$ the reaction was quenched with $n$-hexane. The precipitated proteins were washed, solubilized in $\mathrm{H}_{2} \mathrm{O}$, dialysed to remove residual sulfonic salts, concentrated by lyophilization, and analyzed by SDS-PAGE and anti-SC immunoblots. A diagramatic representation of the weakly staining immunoblot is shown. The bars represent the location of the immunoreactive SC bands in the gel. Incubation of bile (closed bars) and intestinal secretions (slashed bars) with TFMS resulted in a comparable decrease in size of both biliary and intestinal SC, as well as considerable size heterogeneity of the resultant SC (note the greater size range of the bars after TFMS). 
were smaller by comparable amounts. After $180 \mathrm{~min}$ of TFMSmediated deglycosylation, intestinal SC was still about 8,000 mol wt smaller than biliary SC $(64,000$ and $72,000 \mathrm{~mol} \mathrm{wt})$. No decrease in the size of albumin (a nonglycosylated protein) was observed after $180 \mathrm{~min}$ of TFMS treatment (data not shown). Thus, differential glycosylation did not appear to be the cause of the smaller forms of SC found in the jejunum.

Processing of biliary SC by the small intestine. To determine if the smaller size of the jejunal forms of SC was the result of the action of a constituent of jejunal secretions or the jejunal brush border, the fate of biliary SC in the small intestine was examined. Bile collected after an intraperitoneal injection of [ $\left.{ }^{3} \mathrm{H}\right]$ fucose contained four major fucoproteins, including SC (Fig. 5 ); in bile reduced with 2-ME, SC was the largest demonstrable fucopeptide. After incubation of radiolabeled bile in vivo in the isolated loop the amount of radioactivity that could be loaded on the gel was limited because of dilution with jejunal secretions. Nonetheless, in the presence of the protease inhibitors, the 82,000 - and 78,000-mol-wt doublet of biliary SC were greatly reduced and a 70,000-mol-wt radiolabeled band appeared. None of the other radiolabeled biliary proteins were altered significantly in size during the incubation. Since the only band larger than 70,000 mol wt present in the reduced bile was SC, it appeared

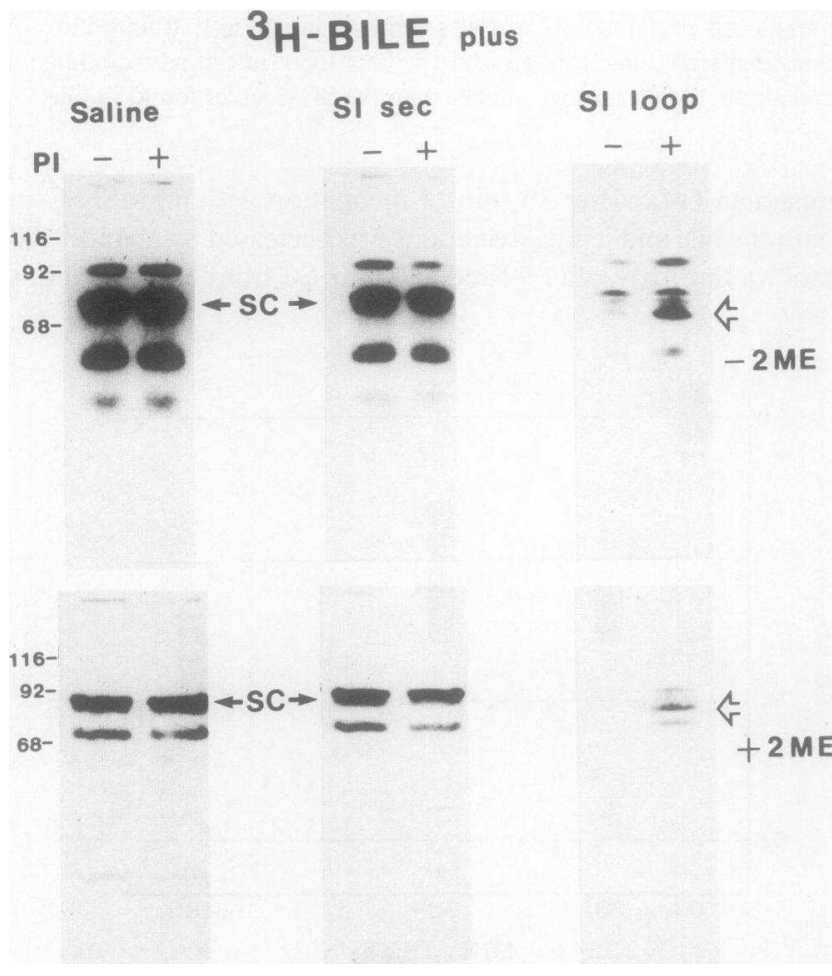

Figure 5. Processing of biliary SC by the small intestine. Bile containing biosynthetically radiolabeled proteins $\left(\left[{ }^{3} \mathrm{H}\right]\right.$ fucose $)$ was incubated in vitro with $150 \mathrm{mM}$ saline or intestinal secretions (SI Sec) in the presence $(+)$ or absence $(-)$ of protease inhibitors, or incubated in vivo in an isolated jejunal loop (SI loop) that had been preperfused with $(+)$ or without $(-)$ protease inhibitors. After $1 \mathrm{~h}$, samples of each incubation were analyzed by SDS-PAGE and radiofluorography under nonreduced (upper panel; -2-ME) or reduced (lower panel; +2-ME) conditions. The location of SC is indicated by the closed arrows. After incubation of radiolabeled bile in the jejunal loop in the presence of protease inhibitors, a 70,000-mol-wt band (open arrows) appeared. This band did not appear after incubation of bile in SI Sec. that biliary SC was degraded to a 70,000-mol-wt form in the jejunal loop. Incubation of radiolabeled bile with jejunal secretions or with $\mathrm{NaCl}$ did not modify the biliary profile or result in the appearance of the 70,000-mol-wt band (Fig. 5). Thus, the jejunal surface, but not the jejunal secretions, was capable of converting the biliary form of SC into a form that was similar to that observed in jejunal secretions. This processing appeared to be specific for SC since none of the other biliary proteins appeared to be degraded.

$B B$-bile mixing experiments. In order to test the possibility that jejunal BB were capable of proteolytically processing SC, biliary SC was incubated with purified jejunal BB membranes (20-fold enriched for sucrase activity with $15 \%$ yield). No SC was identified in the purified BB preparation (Fig. 6). After incubation with $\mathrm{BB}$, the anti-SC immunoblot demonstrated that the biliary SC doublet was partially converted to a 70,000-molwt immunoreactive form. This conversion product could be seen also in the Coomassie-stained gel by the appearance of a 70,000mol-wt protein band in the BB-bile mixture (Fig. 6) that was not present in either the bile or BB alone. The proteolytic activity of the BB membranes was not found in partially purified preparations of jejunal Golgi (ninefold enriched for galactosyl transferase activity with $26 \%$ yield), LBM (17-fold enriched for NaKATPase with $12 \%$ yield), or lysosomes (12-fold enriched for betaglucosaminidase activity with $22 \%$ yield) (data not shown). Thus, jejunal BB appeared to contain a hydrolytic activity that was

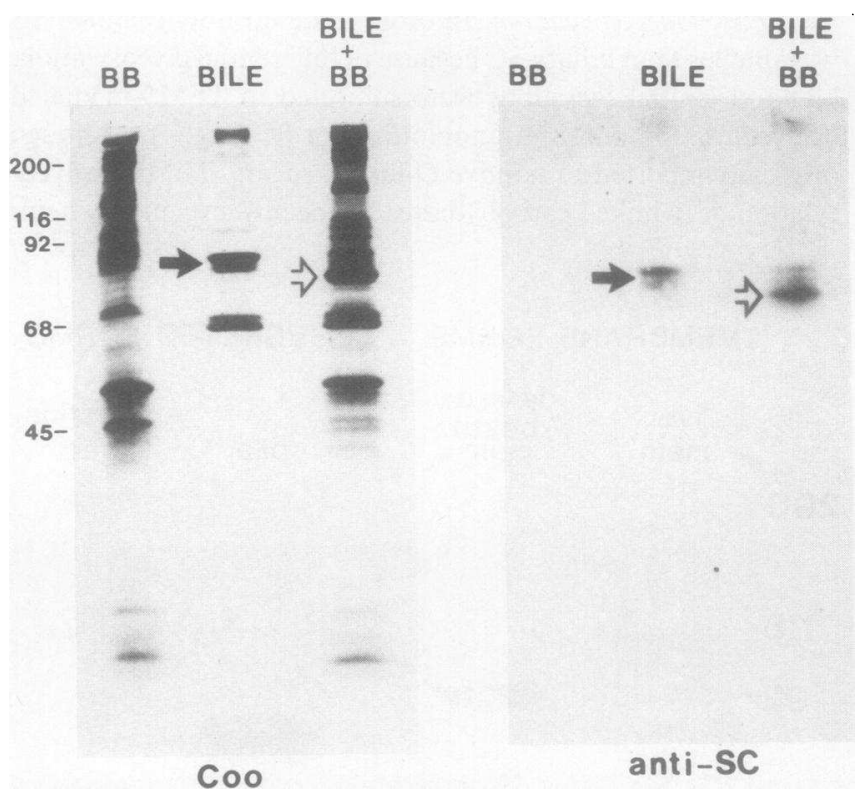

Figure 6. SDS-PAGE and anti-SC immunoblots of BB, bile, and BBbile mixture (bile $+\mathrm{BB}$ ). Partially purified $\mathrm{BB}$ were prepared from rat jejunal mucosa. Bile was collected from the proximal common bile duct. Samples of BB, bile, and a mixture of $50 \mu \mathrm{l}$ of bile plus $50 \mu \mathrm{g}$ of $B B$ were incubated $\left(20^{\circ} \mathrm{C}\right.$ for $\left.30 \mathrm{~min}\right)$, then diluted and boiled in Laemmli sample buffer. One aliquot of each sample was analyzed by SDS-PAGE and stained with Coomassie Blue (Coo). A second aliquot was analyzed by anti-SC immunoblots (anti-SC). The Coomassiestained gel demonstrates the appearance of a 70,000-mol-wt protein band in the bile + BB mixture (open arrow) that was not present in the bile or BB lanes. The anti-SC immunoblot demonstrates that there was no SC detectable in the BB, and that biliary SC (closed arrow) was partially converted to a 70,000-mol-wt protein by incubation with BB (open arrow). 
capable of converting biliary SC to the size of SC found in jejunal secretions. This activity appeared to be specific for SC since other biliary proteins were not affected. The hydrolytic activity was also identified in BB prepared from ileum and renal cortex (data not shown).

Partial characterization of $B B$ hydrolase. Fig. 7 shows that the hydrolysis of biliary SC to the 70,000-mol-wt form by jejunal $\mathrm{BB}$ had a $\mathrm{pH}$ optimum of $\sim 5$. Less efficient hydrolysis $(25-$ $45 \%$ ) occurred, however, over a pH range from 6 to 9. The hydrolytic activity was not present in the $0.5 \mathrm{M} \mathrm{KCl}$ extract but was present in the $0.5 \%$ Triton extract of jejunal BB (Fig. 8).

The hydrolytic activity was not inhibited by the serine protease inhibitors, PMSF, soybean trypsin inhibitor, tosylamidephenyl-ethylchloromethylketone, tosyl-lysine-chloromethylketone, or diisopropylfluorophosphate; nor was it inhibited by the carboxyl protease inhibitor, pepstatin, or the thiol protease inhibitors, $p$-chloromercuriphenyl sulfonic acid or iodoacetamide. However, it was inhibited by the metalloprotease inhibitors 2ME, DTT, and EDTA. No inhibition, however, was observed with the neutral endopeptidase (a BB metalloprotease) inhibitor phosphoramidon. The EDTA-mediated inhibition of the hydrolase (Fig. 9) was partially reversed with the addition of $10^{-6}$ $\mathrm{M} \mathrm{Zn}\left(10^{-4} \mathrm{M} \mathrm{Zn}\right.$ was required for complete reversal of the EDTA-mediated inhibition), whereas $\mathrm{Ca}$ or $\mathrm{Mg}$ was required at a $10^{-3} \mathrm{M}$ concentration before any reversal of inhibition was observed; Co and $\mathrm{Mn}$ failed to reverse the inhibition at any concentration tested $\left(10^{-3}-10^{-6} \mathrm{M}\right)$.

Jejunal membranes and secretions prepared with metalloprotease protection. Jejunal membranes prepared in the presence of metalloprotease inhibitors ( $150 \mathrm{mM} 2-\mathrm{Me}$ and $50 \mathrm{mM}$ EDTA) contained an SC doublet that was of a size comparable to that found in liver membranes (Fig. 10, Membranes). Similarly, jejunal secretions collected in the presence of metalloprotease inhibitors contained an SC form similar in size to biliary SC and was 10,000-15,000 mol wt larger than $S C$ in jejunal secretions collected without metalloprotease inhibitors (Fig. 10, Secretions).

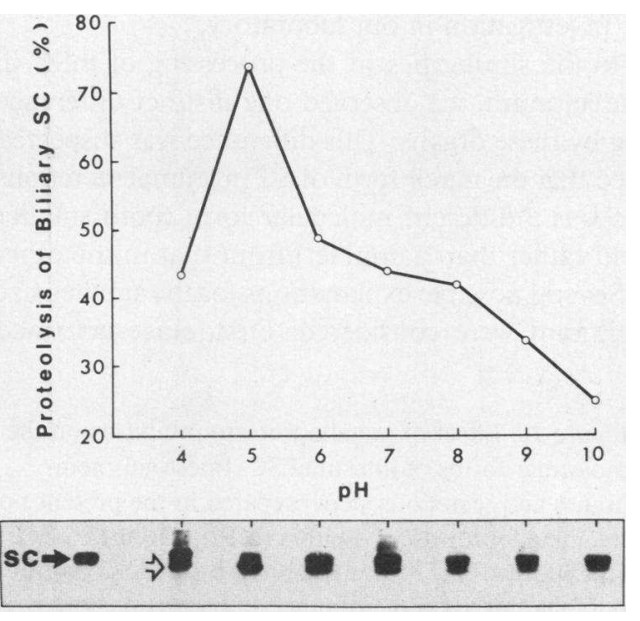

Figure 7. pH dependence of proteolytic conversion of biliary SC by jejunal BB. Incubations of bile and BB were carried out as in Fig. 6, except that the $\mathrm{pH}$ of the mixture was varied. Aliquots of incubations at each $\mathrm{pH}$ were analyzed by anti-SC immunoblots. The percent conversion of biliary SC to the 70,000-mol-wt form was calculated by laser densitometry of the immunoblots. The anti-SC immunoblot in the box shows the $\mathrm{SC}$ bands in the incubation mixtures at each $\mathrm{pH}$ in comparison to biliary SC (closed arrow).

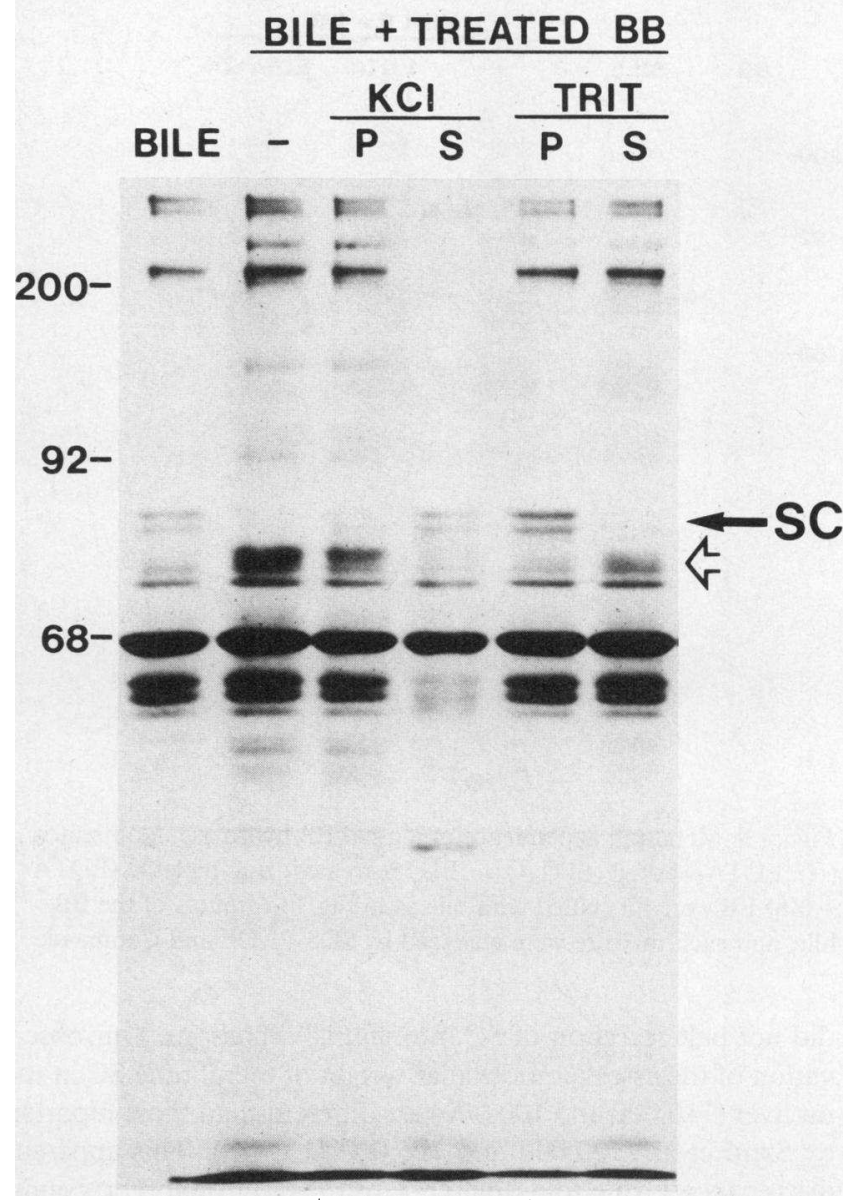

Figure $8 . \mathrm{KCl}$ and Triton (Trit) extraction of $\mathrm{BB}$ hydrolytic activity. BB prepared from rat jejunal mucosa were extracted with $0.5 \mathrm{M} \mathrm{KCl}$ or $0.5 \%$ Triton $\mathrm{X}-100\left(0^{\circ} \mathrm{C}\right.$ for $\left.1 \mathrm{~h}\right)$. The mixtures were centrifuged $(13,000 \mathrm{~g}$ for $20 \mathrm{~min}$ ) and the pellets (P) and supernatants (S) were incubated with bile as in Fig. 6. After incubation, the mixtures were analyzed by SDS-PAGE. Biliary SC $(82,000$ and $78,000 \mathrm{~mol} \mathrm{wt})$ is indicated (closed arrow) in the Coomassie profile of bile in lane 1 (BILE). The 70,000-mol-wt SC band is indicated (open arrow) in the Coomassie profile of a mixture of bile with untreated (-) brush borders. The jejunal hydrolytic activity was assessed by the disappearance of biliary-size SC (closed arrow) and the appearance of the 70,000mol-wt band (open arrow). The hydrolytic activity was present in the pellet of the $\mathrm{KCl}$ extract (KCl-P) and in the supernate of the triton extract (Trit-S).

It should be noted that collection of jejunal secretions with the high concentration of 2-ME was difficult. In some experiments it resulted in dissociation of substantial numbers of enterocytes into the lumen and it consistently distorted the SDS-PAGE and immunoblot profile, as can be seen in Fig. 10 (Secretions).

\section{Discussion}

The results of this study demonstrate that in the jejunum, SC undergoes two sequential posttranslational processing steps. The first step is the conversion of membrane SC to soluble SC by the cleavage of a 20,000-mol-wt membrane-anchoring domain. The second step results in conversion of the bulk of soluble SC from an 80,000- to a 70,000-mol-wt form. These two steps do not appear to be mediated by the same enzyme since inhibition of the BB hydrolase responsible for the second proteolytic step 


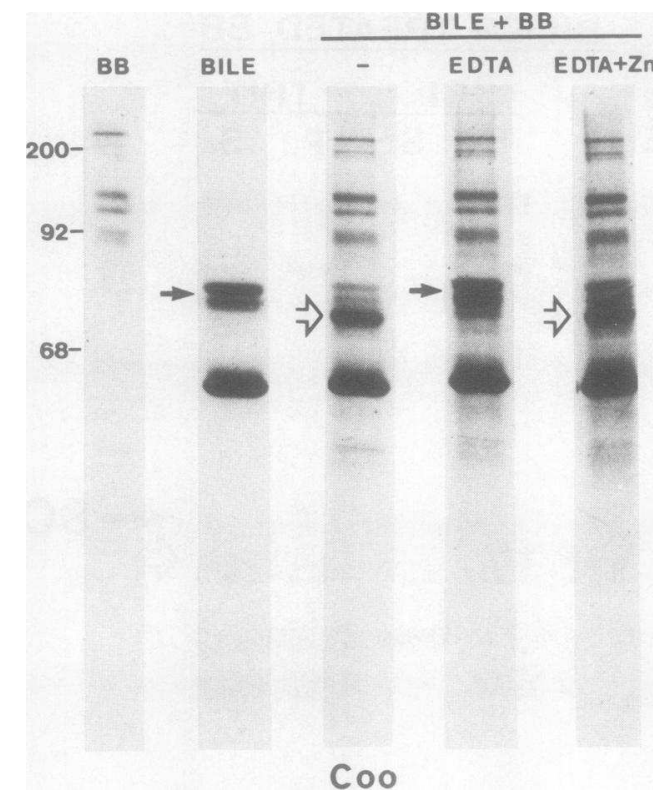

Figure 9. Metal ion dependence of jejunal BB hydrolase. Nontreated (-); EDTA-washed (EDTA); or EDTA-treated, zinc repleted (EDTA $+\mathrm{Zn}$ ) BB were incubated with bile as in Fig. 6. Aliquots of the BB, bile, and each mixture were analyzed by SDS-PAGE and Coomassie

did not halt secretion of SC into jejunal secretions. Our observation of the assessed molecular weight of membrane $\mathrm{SC}$ in the rat liver $(110,000$ and 100,000$)$ are different than those reported by Sztul et al. $(120,000$ and 105,000 [15, 16]). This apparent discrepancy is due to a difference in the electrophoretic conditions. Under reducing conditions (as used by Sztul et al.), our liver membrane SC has an apparent molecular weight of 115,000 and 105,000 .

Solubilization of $\mathrm{mSC}$ by proteolytic cleavage of a membrane anchoring domain has been demonstrated in rabbit mammary gland $(6,7)$, a human colonic cell line $(4)$, and rat liver $(8,15$, 16). Our results not only suggest that a similar proteolytic solubilization step occurs in the rat jejunum but that they also provide additional information about the solubilization step. The finding of a similar 20,000-mol-wt size difference between $\mathrm{mSC}$ and secreted SC in both the small intestine and the liver suggests that a similar (or identical) protease is responsible for $\mathrm{SC}$ solubilization in both tissues. The observation that $\mathrm{mSC}$ was present in jejunal LBM but not in BB suggests that cleavage of the anchoring peptide occurs either in the transport vesicle or rapidly enough after fusion of the transport vesicle with the BB

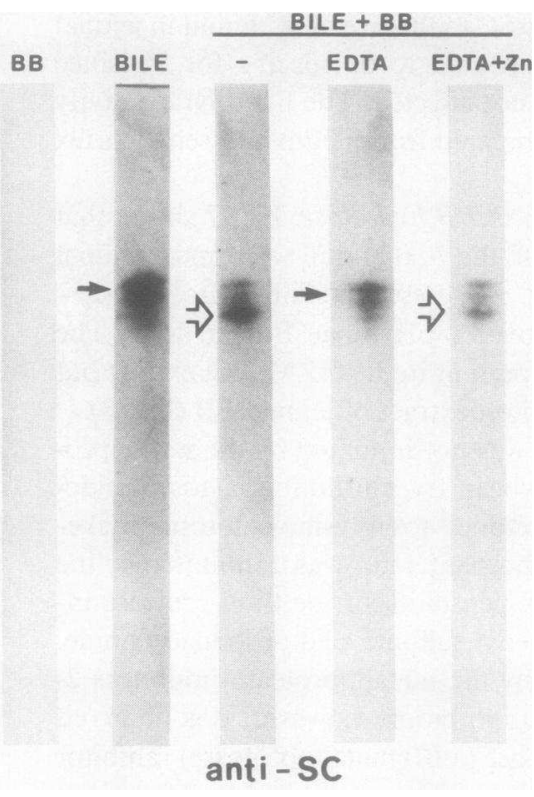

(Coo) stains and by anti-SC immunoblots. The conversion of biliary SC (closed arrows) to the 70,000-mol-wt form (open arrows) was partially prevented by the BB washed with EDTA. $\mathrm{Zn}$ repletion partially restored the hydrolytic activity.

to prevent membrane SC from accumulating in the BB. Our observations are in agreement with electron microscopic evidence from our laboratory that localized SC on the LBM but not on the BB of rat jejunum (3). The finding of Solari and Kraehenbuhl (7) that the membrane anchoring domain of SC is present in the luminal membrane of mammary gland epithelial cells suggests that if the cleavage occurs in the transport vesicle both portions of the cleaved molecule reach the luminal membrane. In the rat liver, both immunoelectron microscopic (17) and cell fractionation data $(15,16,18)$ suggest that solubilization occurs, at least in part, on the canalicular membrane. The precise site of cleavage of membrane to soluble SC in the intestine is currently under investigation in our laboratory.

In contrast to the similarities in the processing of $\mathrm{mSC}$ in the liver and the jejunum, we observed one distinct difference in SC processing by these organs. This difference was suspected when we observed that the major form of SC in jejunal secretions and membranes was a different molecular form (both smaller and a single band rather than a doublet) from that in the other organs studied. Several possible explanations for the smaller size of SC in the jejunum were considered. First, since activated

\section{MEMBRANES}

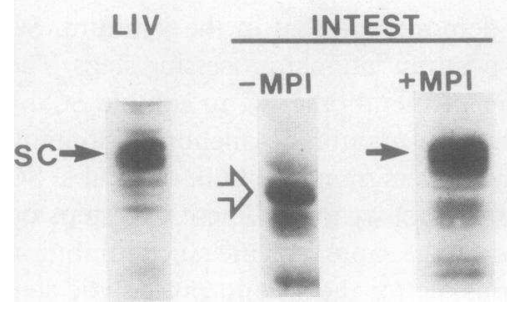

\section{SECRETIONS}

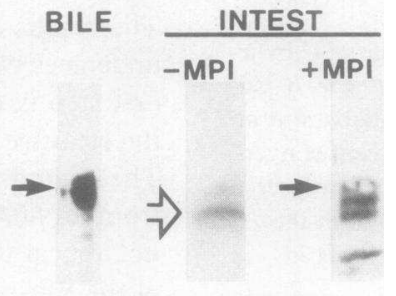

Figure 10. Effect of metalloprotease inhibitors on the molecular forms of intestinal SC. Intestinal membranes and secretions were prepared in the presence of the metalloprotease inhibitors (MPI), $150 \mathrm{mM}$ 2-ME and $50 \mathrm{mM}$ EDTA, and analyzed by anti-SC immunoblots. Intestinal membranes and secretions prepared with metalloprotease inhibitors contained SC that is similar in size to that found in liver (LIV) and bile, respectively (closed arrows), and 10,000-20,000 mol wt larger than SC prepared in the absence of metalloprotease inhibitors (open arrows). The distortion of the immunoblot of SC in jejunal secretions is due to the high concentration of EDTA and 2-ME and the low $\mathrm{SC}$ concentration in the sample. 
pancreatic proteases are present in high concentration in the jejunum but not in the other tissues, they could be responsible for proteolytic cleavage of SC in the jejunum. This possibility was excluded, however, since SC in secretions from a loop of jejunum that had been isolated from pancreatic secretions for 4 wk was still a single 70,000-mol-wt protein. Second, since SC is known to be a glycoprotein (19), it was possible that differential glycosylation (or differential glycosidase activity) explains the smaller size of jejunal SC. This possibility was proven to be unlikely, however, since TFMS-mediated deglycosylation resulted in comparable decreases in size of the SC in bile and jejunal secretions. These results, however, do not exclude the possibility that minor glycosylation differences may exist between liver and jejunal SC.

Third, it was possible that the smaller size of jejunal SC is the result of a posttranslational proteolytic processing step in the jejunal brush border. Several pieces of evidence were obtained that support this mechanism as the correct explanation. (a) Radiolabeled biliary SC was converted from an 82,000- and 78,000mol-wt doublet to a 70,000-mol-wt form during incubation in the rat jejunum. Since the hydrolysis did not occur when bile was incubated with jejunal secretions, the jejunal BB was implicated. (b) Biliary SC was partially converted to the smaller size by incubation with purified jejunal brush borders. The proteolytic activity was present in high activity in brush borders but could not be identified in jejunal LBM, Golgi, or lysosomal membrane populations. (c) SC in jejunal membranes prepared in the presence of metalloprotease inhibitors, which inhibited the BB hydrolytic activity, was the same size as SC found in the other organs. (d) Although it was difficult to collect and analyze jejunal secretions prepared in the presence of metalloprotease inhibitors, these experiments also suggested that the BB metalloprotease was responsible for the smaller size of SC in jejunal secretions.

Thus, our data are consistent with the hypothesis that in the intestine, $\mathrm{SC}$ is synthesized as a membrane protein and expressed on the abluminal plasma membrane (LBM) as a protein doublet $(110,000$ and $100,000 \mathrm{~mol} \mathrm{wt})$ comparable to $\mathrm{mSC}$ in the liver. In both tissues, membrane SC is solubilized to an $80,000-\mathrm{mol}$ wt protein by proteolytic cleavage of the ectoplasmic domain from the membrane-anchoring peptide. In the jejunum, however, $\mathrm{SC}$ undergoes a second proteolytic processing step that is mediated by an integral BB protease resulting in the 70,000-molwt major form of SC that is secreted into jejunal fluids. The second proteolytic step may occur during or after secretion of soluble SC into the intestinal lumen. That the protease can act on luminal contents is demonstrated by the conversion of most of the biliary SC to the smaller form by incubation in a jejunal loop. If the hydrolysis occurs after secretion, it must occur rapidly enough to prevent significant accumulation of the 80,000-molwt form in jejunal secretions.

The brush border SC endopeptidase was characterized as an integral membrane metalloprotease with an acid pH optimum. Although it has some similarities to the neutral endopeptidase described by Kerr, Fulcher, and Kenny $(21,22)$, it is in other ways distinct. Both are $\mathrm{Zn}$-dependent metalloproteases, and like the neutral endopeptidase, the SC endopeptidase is also present in renal tubular BB membranes. The SC endopeptidase, however, was not inhibited by phosphoramidon and has an acid $\mathrm{pH}$ optimum, whereas the neutral endopeptidase is inhibited by phosphoramidon and has a neutral $\mathrm{pH}$ optimum. Furthermore, incubation of rat bile with purified preparations of pig kidney neu- tral endopeptidase EC 3.4.24.11 and rat kidney neutral endopeptidase II (kindly performed by Dr. Kenny, University of Leeds, Leeds, United Kingdom) failed to result in proteolytic conversion of biliary SC to the 70,000 -mol-wt form. Thus, the SC endopeptidase appears to be distinct from the previously characterized brush border endopeptidases.

The finding that soluble SC in colonic secretions and in milk is the same size as biliary SC suggests that lumenal membranes of the colon and breast do not contain the SC-endopeptidase. Renal tubular cell microvillar membranes, however, do contain the protease. Thus, the SC-endopeptidase distribution is similar to that of several other intestinal microvillar membrane peptidases, such as the aminopeptidases (20).

The purpose of the hydrolysis of SC to the 70,000-mol-wt form in the small intestine is not clear. It is not essential for secretion of SC because inhibition of the proteolytic step with 2-ME does not prevent SC secretion in the jejunum and SC secretion proceeds in the colon in the absence of the proteolytic step. In the small intestine, the step does not appear to be obligatory in that a small portion of SC in jejunal secretions remains uncleaved (Fig. 1) and a small portion of biliary SC is not converted to the 70,000-mol-wt size in the isolated intestinal loop (Fig. 5). Both free SC and SC complexed with IgA appear to undergo this processing step since the 70,000-mol-wt form of $\mathrm{SC}$ is also the major form found in gels of reduced fluids containing SIg (conditions that would dissociate covalently bound SC from IgA). The processing step likely occurs at the time of, or after, secretion of SC into the lumen since the protease is located strictly in the $\mathrm{BB}$. Consequently, the processing step is unlikely to have a role in the binding of $\mathrm{pIg}$ to $\mathrm{SC}$, which occurs on the abluminal face of the cell, or in the transcellular transport of pIg. The BB hydrolytic activity is quite specific for SC; of all the proteins present in the bile, only SC appeared to be degraded after mixing with BB membranes. Whether this hydrolysis of SC by the jejunal BB protease results in any alteration in SC function remains to be determined.

The finding of a doublet of SC in rat liver membranes and bile is consistent with previous observations from our (8) and other laboratories $(9,16)$. Sztul et al. (16) observed three molecular forms of SC: an intracellular 105,000-mol-wt precursor that was converted to a 116,000-mol-wt form in the Golgi and subsequently converted to a 120,000 -mol-wt form at the plasma membrane. The explanation for the presence of two soluble SC bands is not yet established; carbohydrate heterogeneity or proteolysis seem the most likely explanations. Both genetic and carbohydrate heterogeneity have been reported to account for the multiple molecular forms of SC found in the rabbit $(5,6$, 23). The observation that the biliary doublet is converted by the BB hydrolase to a single 70,000-mol-wt immunoreactive band suggests that if carbohydrate heterogeneity accounts for the SC doublet, the region containing the carbohydrate differences is removed by the $\mathrm{BB}$ protease.

In summary, we have demonstrated that in the rat jejunum SC undergoes two posttranslational processing steps. The first step is the conversion of membrane SC to soluble SC by cleavage of the membrane-anchoring domain. This solubilization occurs by a mechanism similar to that for solubilization of SC in the liver and other glandular organs. The second step occurs only in the jejunum and results in cleavage of an additional 10,000mol-wt portion from SC. The second posttranslational processing step is mediated by a specific protease present in jejunal brush border membranes. SC is the only endogenous substrate iden- 
tified for the protease. The SC-endopeptidase is an integral BB metalloprotease with an acidic $\mathrm{pH}$ optimum and appears to be distinct from any previously described jejunal BB protease.

\section{Acknowledgments}

The authors would like to express appreciation to Dr. W. R. Brown for his critical review of this work, Ms. Kathryn Campbell and Ms. Doris Gaskin for technical assistance, and Ms. Jean Gilbert for expert secretarial support.

This work was supported by the Veterans Administration and by grants AM33664 and BRSG0537 from the National Institutes of Health. Dr. Ahnen is the recipient of a Veterans Administration Research Associate Award (No. 1312).

\section{References}

1. Singleton, J. D., D. J. Ahnen, and T. M. Kloppel. 1984. Sorting and post-translational processing of secretory component by the intestinal epithelial cell. J. Cell Biol. 99(4, Pt. 2):281a. (Abstr.)

2. Hoops, T. C., T. M. Kloppel, and D. J. Ahnen. 1985. Characterization of the brush border protease that mediates the post-translational processing of secretory component in the rat enterocyte. Gastroenterology. 88:1422. (Abstr.)

3. Brown, W. R., Y. Isobe, and P. K. Nakane. 1976. Studies on the translocation of immunoglobulins across intestinal epithelium. II. Immunoelectron microscopic localization of immunoglobulins and secretory component in human intestinal mucosa. Gastroenterology. 71:985-995.

4. Mostov, K. E., and G. Blobel. 1982. A transmembrane precursor of secretory component: the receptor for transcellular transport of polymeric immunoglobulins. J. Biol. Chem. 257:11816-11821.

5. Knight, K. L., M. Rosensweig, E. A. Lichter, and W. C. Hanly. 1974. Rabbit secretory IgA: identification and genetic control of two allotypes of secretory component. J. Immunol. 112:877-882.

6. Mostov, K. E., J. P. Kraehenbuhl, and G. Blobel. 1980. Receptormediated transcellular transport of immunoglobulin: synthesis of secretory component as multiple and larger transmembrane forms. Proc. Natl. Acad. Sci. USA. 77:7257-7261.

7. Solari, R., and J. P. Kraehenbuhl. 1984. Biosynthesis of the IgA antibody receptor: a model for the transepithelial sorting of a membrane glycoprotein. Cell. 36:61-71.

8. Kloppel, T. M., and W. R. Brown. 1984. Rat liver membrane secretory component is larger than free secretory component in bile. Evidence of proteolytic conversion of the membrane form to the free form. J. Cell. Biochem. 24:307-318.

9. Sztul, E. S., K. E. Howell, and G. E. Palade. 1983. Intracellular and transcellular transport of secretory component and albumin in rat hepatocytes. J. Cell Biol. 97:1582-1591.

10. Ahnen, D. J., A. K. Mircheff, N. A. Santiago, C. Yoshioka, and G. M. Gray. 1983. Intestinal surface aminooligopeptidase: distinct molecular forms during assembly on intracellular membranes in vivo. $J$. Biol. Chem. 258:5960-5966.

11. Ahnen, D. J., N. A. Santiago, J. P. Cezard, and G. M. Gray. 1982. Intestinal aminooligopeptidase: in vivo synthesis on intracellular membranes of rat jejunum. J. Biol. Chem. 257:12129-12135.

12. Edge, A. S., C. R. Faltynek, H. Liselotte, L. E. Reichert, Jr., and P. Weber. 1981. Deglycosylation of glycoproteins by trifluoromethane sulfonic acid. Anal. Biochem. 118:131-137.

13. Laemmli, U. K. 1970. Cleavage of structural proteins during the assembly of the head of bacteriophage $T_{4}$. Nature (Lond.). 227:680-685.

14. Stewart, J. R., M. F. Chaplin, and A. J. Kenny. 1984. Deglycosylation by trifluoromethanesulphonic acid of endopeptidase-24.11 purified from pig kidney and intestine. Biochem. J. 221:919-922.

15. Sztul, E. S., K. E. Howell, and G. E. Palade. 1985. Biogenesis of the polymeric IgA receptor in rat hepatocytes. I. Kinetic studies of its intracellular forms. J. Cell Biol. 100:1248-1254.

16. Sztul, E. S., K. E. Howell, and G. E. Palade. 1985. Biogenesis of the polymeric IgA receptor in rat hepatocytes. II. Localization of its intracellular forms by cell fractionation studies. J. Cell Biol. 100:12551261.

17. Takahashi, I., P. K. Nakane, and W. R. Brown. 1982. Ultrastructural events in the translocation of polymeric IgA by rat hepatocytes. $J$. Immunol. 128:1181-1187.

18. Meier, P. J., E. S. Sztul, A. Reuben, and J. L. Boyer. 1984. Structural and functional polarity of canalicular and basolateral plasma membrane vesicles isolated in high yield from rat liver. J. Cell Biol. 98:9911000 .

19. Mizoguchi, A., T. Mizuochi, and A. Kobata. 1982. Structure of the carbohydrate moieties of secretory component purified from human milk. J. Biol. Chem. 257:9612-9621.

20. Desnuelle, P. 1979. The tenth Sir Hans Krebs lecture. Intestinal and renal aminopeptidase: a model of a transmembrane protein. Eur. J. Biochem. 101:1-11.

21. Kerr, M. A., and A. J. Kenny. 1974. The molecular weight and properties of a neutral metallo-endopeptidase from rabbit kidney brush border. Biochem. J. 137:489-495.

22. Fulcher, I. S., and A. J. Kenny. 1983. Proteins of the kidney microvillar membrane. Biochem. J. 211:743-753.

23. Kuhn, L. C., H. P. Kocher, W. C. Hanly, L. Cook, J. C. Jaton, and J. P. Kraehenbuhl. 1983. Structural and genetic heterogeneity of the receptor mediating translocation of immunoglobulin $\mathrm{A}$ dimer antibodies across epithelia in the rabbit. J. Biol. Chem. 258:6653-6659. 